\title{
Phase Separation in two-Species Atomic Bose-Einstein Condensate with Interspecies Feshbach Resonance
}

\author{
Lu Zhou ${ }^{1}$, Jing Qian ${ }^{1}$, Han $\mathrm{Pu}^{2, * *}$, Weiping Zhang ${ }^{1, \dagger}$, and Hong Y. Ling ${ }^{3, *}$ \\ ${ }^{1}$ State Key Laboratory of Precision Spectroscopy, Department of Physics, \\ East China Normal University, Shanghai 200062, China \\ ${ }^{2}$ Department of Physics and Astronomy, and Rice Quantum Institute, \\ Rice University, Houston, TX 77251-1892, USA and \\ ${ }^{3}$ Department of Physics and Astronomy, Rowan University, Glassboro, New Jersey 08028-1700, USA
}

\begin{abstract}
We consider a mixture of two-species atomic Bose-Einstein codensates coupled to a bound molecular state at zero temperature via interspecies Feshbach resonance. The interspecies Feshbach coupling precludes the possibility of doubly mixed phases while enables not only the pure molecular superfluid but also the pure atomic superfluids to exist as distinct phases. We show that this system is able to support a rich set of phase separations, including that between two distinct mixed atom-molecule phases. We pay particular attention to the effects of the Feschbach coupling and the particle collisions on the miscibility of this multi-component condensate system.
\end{abstract}

PACS numbers: 03.75.Mn, 05.30.Jp

\section{INTRODUCTION}

The experimental realization of multi-component condensate systems in the late-90s [1, 2, 3] has renewed the interest in the subject of miscibility of two distinguishable condensates in a binary mixture [4, 5, 6, 7, 8]. Whether two condensates coexist in the same spatial volume (miscibility) or repel each other into separate spatial regions (immiscibility or phase separation) or even collapse when they are brought together is largely determined by the relative strength between inter- and intra-species s-wave scattering lengths. The ability to change the two-body interactions by tuning the magnetic field across the Feshbach resonance in atomic systems has enabled the observation of phase separations, that are otherwise impossible with traditional condensed matter environments, to be accessed experimentally. The recent experimental demonstration of rich phase structure including the phase separation in an unbalanced fermionic superfluidity system [9, 10] serves as a great testament to the remarkable controllability that the technique of Feshbach resonance can bring to the atomic system.

In this paper, we consider a model where bosonic atoms of two different species are coupled to a bound heteronuclear molecular state via the interspecies Feshbach resonance (we will simply refer to it as the heteronuclear model in this paper). Chiefly due to their large permanent electric dipole moments, ultracold heteronuclear molecules are thought to hold great promise in applications such as quantum computing and simulation [11, 12], and precision measurement of fundamental symmetry [13, 14, 15] and constants [16, 17, 18]. As such, the study of cold heteronuclear molecules has evolved into one of the most hotly pursued researches in the field of atomic, molecular and optical physics [19]. The recent experimental efforts both in achieving the tunable interaction between ${ }^{41} \mathrm{~K}$ and ${ }^{87} \mathrm{Rb}$ [20] and in demonstrating the ability of the Feshbach resonance to control the misci- bility of a ${ }^{85} \mathrm{Rb}^{-}{ }^{87} \mathrm{Rb}$ dual-species BEC $[21]$ have further stimulated our interest in the proposed model.

In an earlier paper 22], we have concentrated on the coherent creation of heteronuclear molecular condensates (similar studies can also be found in reference [23]). In this paper, we will focus on constructing zerotemperature phase diagrams, paying particular attentions to phase separation and collapse. In models with a broad Feshbach resonance, the molecular component can be adiabatically eliminated; this approximation leads to an effective binary condensate system, whose properties were investigated extensively in the past $[4,5,6,6,7,8]$. In general, we must treat the molecular component as an independent degree of freedom, regarding our model as a genuine three-species condensate system. However, our system is fundamentally different from the independent gas model 24, 25], where no interspecies conversions can take place. The conversion between atoms and molecules can lead to phenomena unconventional to the independent gas model, as has been pointed out by Radzihovsky et al. [26] and Romans et al. 27]. The surprise, that the homonuclear model admits the molecular superfluid (MSF) but not the pure atomic superfluid (ASF), was traced to the intraspecies Feshbach process which takes two atoms from the same species to form one molecule. This is no longer the case for the interspecies Feshbach process which requires only one atom from each species to produce one molecule. But, it creates a new surprise - the doubly mixed phases with two nonvanishing order parameters which are ubiquitous in the independent gas model are completely absent from our system. For the same reason, not only the pure molecular phase but also the pure atomic superfluids can exist in the heteronuclear model. This along with the possibility of simultaneously tuning the Feshbach energy and the atomic population imbalance (an extra control "knob" unique to the heteronuclear model) makes the number of experimentally accessible phase separations (summarized in Sec. VI) far greater in the heteronuclear model than in the homonu- 
clear model.

This paper is organized as follows. In Sec. II, we describe our model and the Hamiltonian by defining all the system parameters. In Sec. III, we derive the equilibrium conditions by minimizing a total grand canonical Hamiltonian under the premise that the system is in an arbitrary state of phase separation. A general classification of various phases supported by our system is summarized in Sec. IV. The same section also presents the nonlinear coupled equation governing the mixed atommolecule superfluid phase, and generalize the stability conditions to our three-component system. In Sec. V, we provide numerical examples that showcase the ability of our system to support a rich set of phase separation possibilities while at the same time elucidate the effects of the Feshbach coupling and the particle collisions on the miscibility property of our system. Finally, a summary is given in Sec. VI.

\section{MODEL AND HAMILTONIAN}

Our model consists of two distinct atomic species of state $|1\rangle$ and $|2\rangle$, and a molecular species of state $|3\rangle$. The state $|3\rangle$ differs in energy from the atomic states by an amount $\delta$. In our model, particles can interact with each other through collisions. At low temperature, we use $\chi_{i j}$ to denote the collisional strength of the swave scattering involving either same species $(i=j)$ or different species $(i \neq j)$. In addition, atoms of state $|1\rangle$ can combine with atoms of state $|2\rangle$ to coherently create molecules of state $|3\rangle$ via Feshbach resonance characterized with atom-molecule coupling strength $g$ and Feshbach detuning $\delta$, tunable by changing the magnetic field. Let $\hat{\Psi}_{i}(\mathbf{r})$ be the field operator for the $i$-th component, and $m_{i}$ be the mass of species $i$ with $m_{3}=m_{1}+m_{2}$. We describe our system with the following Hamiltonian

$$
\begin{aligned}
\hat{H}= & \int d^{3} \mathbf{r}\left\{\sum_{i=1,2,3} \hat{\Psi}_{i}^{\dagger}(\mathbf{r})\left(-\frac{\hbar^{2} \nabla^{2}}{2 m_{i}}\right) \hat{\Psi}_{i}(\mathbf{r})\right. \\
& +\delta \hat{\Psi}_{3}^{\dagger}(\mathbf{r}) \hat{\Psi}_{3}(\mathbf{r})+\frac{g}{2}\left[\hat{\Psi}_{3}^{\dagger}(\mathbf{r}) \hat{\Psi}_{1}(\mathbf{r}) \hat{\Psi}_{2}(\mathbf{r})+h . c\right] \\
& \left.+\sum_{i, j} \frac{\chi_{i j}}{2} \hat{\Psi}_{i}^{\dagger}(\mathbf{r}) \hat{\Psi}_{j}^{\dagger}(\mathbf{r}) \hat{\Psi}_{j}(\mathbf{r}) \hat{\Psi}_{i}(\mathbf{r})\right\}
\end{aligned}
$$

which can be easily generalized from the Hamiltonian for the homonuclear model [26, 27].

In this paper, we limit our study to the homogeneous condensate system at zero-temperature where each species has already condensed into the zero momentum mode. As such, we can describe such a system in the spirit of the mean-field approximation in which $\hat{\Psi}_{i}(\mathbf{r})$ is replaced with the uniform $c$-number $\psi_{i} \exp \left(i \varphi_{i}\right)$, where $\psi_{i}>0$ and $\varphi_{i}$ are two real numbers, representing, respectively, the modulus and the phase of the corresponding complex order parameter. It can be easily shown that the mean-field energy density $\mathcal{E}=\langle\hat{H}\rangle / V$ (with $V$ being the total volume) is a function of the "relative phase" $\varphi=\varphi_{1}+\varphi_{2}-\varphi_{3}$ and is minimized when $\varphi=\pi$ (assuming, without loss of generality, that $g>0$ ). With these considerations, we derive from Eq. (1) the following mean-field (low branch) energy density

$$
\mathcal{E}=\delta \psi_{3}^{2}-g \psi_{1} \psi_{2} \psi_{3}+\sum_{i j} \frac{\chi_{i j}}{2} \psi_{i}^{2} \psi_{j}^{2},
$$

which will be used in the next section as the starting point for studying phase and phase separations.

\section{GENERAL CONDITIONS FOR PHASE SEPARATION}

To begin with, we imagine that our system at the ground state is phase separated into a number of arbitrary phases (yet to be determined), each of which occupying a volume of $V_{i}$. For the purpose of easy illustration, without loss of generality, we consider the case that the system separates into two different phases. The total energy of the system, when the interface energies are ignored [5, 8], then becomes

$$
E_{\text {total }}=V_{1} \mathcal{E}^{(1)}+V_{2} \mathcal{E}^{(2)}
$$

where $\mathcal{E}^{(n)}$ is the mean-field energy density of the $n$th phase and is equivalent to $\mathcal{E}$ in Eq. (2) when $\psi_{i}$ is replaced with $\psi_{i}^{(n)}$, the order parameter of the $i$ th component in the $n$th phase. The ground state energy without phase separation is then a special case where, for example, $V_{2}=0$ and $V_{1}=V$.

To determine the ground state, we construct a generalized (mean-field) grand canonical Hamiltonian

$$
K_{\text {total }}=E_{\text {total }}-\mu N-h N_{d}-m V
$$

where $\mu, h$, and $m$ are three Lagrangian multipliers associated, respectively, with the conservation of the total particle number

$$
\sum_{n=1}^{2}\left[\left(\psi_{1}^{(n)}\right)^{2}+\left(\psi_{2}^{(n)}\right)^{2}+2\left(\psi_{3}^{(n)}\right)^{2}\right] V_{n}=N
$$

the total atom number difference

$$
\sum_{n=1}^{2}\left[\left(\psi_{1}^{(n)}\right)^{2}-\left(\psi_{2}^{(n)}\right)^{2}\right] V_{n}=N_{d}
$$

and finally the total volume

$$
V_{1}+V_{2}=V
$$

To proceed further, we first define the free energy density

$$
\mathcal{K}=-\sum_{i} \mu_{i} \psi_{i}^{2}-g \psi_{1} \psi_{2} \psi_{3}+\sum_{i j} \frac{\chi_{i j}}{2} \psi_{i}^{2} \psi_{j}^{2}
$$


When the use of Eqs. (3), (5), and (7) is made, we find that

$$
K_{\text {total }}=V_{1} \mathcal{K}^{(1)}+V_{2} \mathcal{K}^{(2)}-m\left(V_{1}+V_{2}\right),
$$

where $\mathcal{K}^{(n)}$ is equivalent to $\mathcal{K}$ in Eq. (8) with $\psi_{i}$ and $\mu_{i}$ substituted with $\psi_{i}^{(n)}$ and $\mu_{i}^{(n)}$, respectively. In Eq. (8),

$$
\mu_{i}^{(1)}=\mu_{i}^{(2)}=\mu_{i},
$$

with $\mu_{1}=\mu+h, \mu_{2}=\mu-h$, and $\mu_{3}=2 \mu-\delta$. Thus, as expected, the chemical potentials in equilibrium are balanced as a consequence of the particle number conservation laws [Eqs. (5) and (6)]. Minimization of $K_{\text {total }}$ with respect to $V_{1}$ and $V_{2}$ results in

$$
\mathcal{K}^{(1)}=\mathcal{K}^{(2)} \text {. }
$$

Thus, we conclude that (1) for such a phase separation to be possible, phase 1 and phase 2 must coexist for a given set of $\mu_{1}, \mu_{2}$ and $\mu_{3}$ or must overlap in the chemical potential space, and (2) phase separation takes place along the first-order phase transition line where the energy density of phase 1 equals that of phase 2 28]. Evidently, the same conclusion holds for phase separation involving an arbitrary number of phases.

\section{POSSIBLE PHASES AND STABILITY CRITERIA}

\section{A. Possible Phases}

In order to determine the possible phases, we minimize Eq. (9) with respect to $\psi_{i}^{(n)}$. This procedure results in the following set of saddle-point equations,

$$
\begin{aligned}
\mu_{1} \psi_{1} & =-\frac{g}{2} \psi_{2} \psi_{3}+\psi_{1} \sum_{i} \chi_{1 i} n_{i}, \\
\mu_{2} \psi_{2} & =-\frac{g}{2} \psi_{1} \psi_{3}+\psi_{2} \sum_{i} \chi_{2 i} n_{i}, \\
\mu_{3} \psi_{3} & =-\frac{g}{2} \psi_{1} \psi_{2}+\psi_{3} \sum_{i} \chi_{3 i} n_{i} .
\end{aligned}
$$

where we have replaced $\psi_{i}^{(n)}$ with $\psi_{i}$ and used $n_{i}=\psi_{i}^{2}$ to denote the density of the $i$ th species. The possible phases, which correspond to different solutions of Eqs. (11), can then be broadly divided into five groups:

(i) Vacuum with $\psi_{1}=\psi_{2}=\psi_{3}=0$;

(ii) Atomic superfluid of species 1 (ASF1) with $\psi_{1}=$ $\sqrt{\mu_{1} / \chi_{11}}, \psi_{2}=\psi_{3}=0$;

(iii) Atomic superfluid of species 2 (ASF2) with $\psi_{2}=$ $\sqrt{\mu_{2} / \chi_{22}}, \psi_{1}=\psi_{3}=0$;

(iv) Molecular superfluid (MSF) with $\psi_{3}=\sqrt{\mu_{3} / \chi_{33}}$, $\psi_{1}=\psi_{2}=0$;

(v) Mixed atom-molecule superfluid (AMSF) with $\psi_{1} \neq 0, \psi_{2} \neq 0$ and $\psi_{3} \neq 0$.
We first note that our model allows the existence of both MSF and ASF, in contrast to the homonuclear model, which supports MSF but not ASF. In our model, the energy density due to the Feshbach coupling is $g \psi_{1} \psi_{2} \psi_{3}$, which is symmetric with respect to the exchange of any pair of states and hence does not favor the formation of one pure phase to another. In the homonuclear model, on the other hand, the energy density due to the Feshbach coupling is in the asymmetric form of $g \psi_{3} \psi_{1}^{2}$. With such a form, condensation of atoms will inevitably lead to the formation of the molecular condensate while condensation of molecules can take place in the absence of the atomic population.

We emphasize that this classification holds only in the presence of the Feshbach coupling $(g \neq 0)$. In a typical three interacting gas system where $g=0$, the system supports, besides all the phases listed above, doubly mixed phases, for example, ASF1-MSF in which $\psi_{1} \neq 0$, $\psi_{3} \neq 0$, and $\psi_{2}=0$. The absence of the doubly mixed phases can again be attributed to the unique form of the Feshbach coupling, $g \psi_{1} \psi_{2} \psi_{3}$, in the heteronuclear model. This term dictates that the existence of condensate population in any pair of states will lead to the condensation in the third component, which precludes the possibility of forming the doubly mixed phases where the population in the third component is zero.

We also comment that for a given set of chemical potentials, while ASF1, ASF2, and MSF are unique, AMSF, due to the highly nonlinear nature of Eq. (11), may itself be divided into different types depending on the density distribution among three species. Such an example will be given in Sec. $\mathrm{VC} 2$

Finally, this classification, although obtained under the condition of zero temperature, can provide a useful road map for understanding the phase diagrams at finite temperature. At finite temperature, state (i) becomes the so-called normal state where the gas mixture does not possess any order, and other states become mixed in the sense that superfluidities and thermal densities are both present. Lowering temperature reduces the entropy and restores the order to the gas system, thereby giving rise to a hierarchy of critical temperatures associated with phase transitions that break symmetries at various levels. The subject of finite temperature phase diagrams will be left as a future study.

\section{B. Stability Criteria}

From Eq. (11) we have determined the possible phases, which correspond to the critical points (extrema or saddle points) of the free energy density $\mathcal{K}$. We now turn our attention to studying their stability properties by identifying the local minimum of the function $\mathcal{K}$. For this purpose, we first introduce the Hessian matrix

$$
\text { Hessian }=\left(\begin{array}{lll}
f_{11} & f_{12} & f_{13} \\
f_{12} & f_{22} & f_{23} \\
f_{13} & f_{23} & f_{33}
\end{array}\right),
$$


where the matrix element is defined as $f_{i j}=\partial_{\psi_{i}} \partial_{\psi_{j}} \mathcal{K}$, which, with the help of Eq. (8), are shown to have the form

$$
\begin{gathered}
f_{12}=-g \psi_{3}+4 \chi_{12} \psi_{1} \psi_{2}, \\
f_{13}=-g \psi_{2}+4 \chi_{13} \psi_{1} \psi_{3}, \\
f_{23}=-g \psi_{1}+4 \chi_{23} \psi_{2} \psi_{3}, \\
f_{i i}=4 \chi_{i i} n_{i}+2 \sum_{j=1}^{3} \chi_{i j} n_{j}-2 \mu_{i} .
\end{gathered}
$$

Note that we have defined the partial derivatives in the Hessian matrix to be taken with respect to $\psi_{i}$ instead of $n_{i}$ so that we can carry out the stability analysis of both pure and mixed phases in a unified manner.

A state (phase) is thermodynamically stable if it is a local minimum of the function $\mathcal{K}$ for a given set of chemical potentials. This requires the Hessian matrix to be positive semidefinite, which, for a symmetric matrix as in Eq. (12), is possible if and only if all the principal minors of Hessian matrix are nonnegative. This leads to a hierarchy of stability conditions

$$
\begin{gathered}
f_{i i} \geq 0, \\
f_{i i} f_{j j}-f_{i j}^{2} \geq 0, \quad(i \neq j), \\
f_{11} f_{22} f_{33}+2 f_{12} f_{13} f_{23} \\
-f_{11} f_{23}^{2}-f_{22} f_{13}^{2}-f_{33} f_{12}^{2} \geq 0,
\end{gathered}
$$

which reflects the underling philosophy that for a threecomponent miscible system to be stable, all the subsets with single, double, and triple components must also independently satisfy the relevant stability conditions [24].

\section{RESULTS AND DISCUSSIONS}

In this section, we construct phase diagrams and discuss phase separations under a variety of conditions. In all the numerical examples, we use two quantities, $n_{0}$ with the dimension of the density and $\mathcal{E}_{0}=$ $\hbar^{2} n_{0}^{2 / 3}\left(m_{1}+m_{2}\right) / m_{1} m_{2}$ with the dimension of the energy, to define a scaling which consists of the following set of dimensionless variables: $\overline{\mathcal{K}}=\mathcal{K} / \mathcal{E}_{0} n_{0}, \bar{n}_{i}=n_{i} / n_{0}$, $\bar{\psi}_{i}=\psi_{i} / \sqrt{n_{0}}, \bar{\mu}_{i}=\mu_{i} / \mathcal{E}_{0}, \bar{\delta}=\delta / \mathcal{E}_{0}, \bar{g}=g \sqrt{n_{0}} / \mathcal{E}_{0}$, and $\bar{\chi}_{i j}=\chi_{i j} n_{0} / \mathcal{E}_{0}$. To gain a familiarity with the parameters, we consider the interspecies Feshbach resonance around $35 G$ in the ${ }^{41} K-{ }^{87} R b$ mixture, which is characterized with a magnetic field width $\Delta B=5.1 G$, a magnetic moment difference $\Delta \mu=0.005 \mu_{B}\left(\mu_{B}\right.$ being the Bohr magneton), and a background scattering length $a_{b g}=284 a_{0}$ ( $a_{0}$ being the Bohr radius) [20, 29]. For a typical density $n_{0}=10^{15} \mathrm{~cm}^{-3}$, the energy unit is around $\mathcal{E}_{0} \approx 2.4 \times 10^{-29} \mathrm{~J}$. Thus, $g=\sqrt{2 \pi \hbar^{2} a_{b g} \Delta B \Delta \mu / m_{12}}$, when converted into the unitless form, becomes $\bar{g} \approx 0.96$. Additionally, based on $a_{11}=99 a_{0}, a_{22}=60 a_{0}$, and $a_{12}=$ $640 a_{0}$ [29] where we have designated ${ }^{87} R b$ as species 1 and ${ }^{41} K$ as species 2 , we estimate that $\bar{\chi}_{11} \approx 0.2, \bar{\chi}_{22} \approx 0.3$, and $\bar{\chi}_{12} \approx 2.13$.
In our discussions below, we first map out the phase diagrams in the chemical potential space. This includes solving Eqs. (11) to obtain different phases and to perform stability analysis according to the criteria (13). These diagrams enable us to identify the regions where different phases overlap and hence help to answer the question of into which phases an unstable homogeneous system will separate. However, from the experimental point of view, more useful are the phase diagrams in the $\delta$ - $d\left(\equiv n_{1}-n_{2}\right)$ space, which are directly accessible to experiments via two control "knobs", the Feshbach detuning $\delta$ and the two species atomic condensate population difference $d$.

\section{A. Collisionless Limit}

In order to identify the roles of collisions more clearly in later sections, we first consider a situations where we artificially turn off all the $s$-wave scatterings. For pedagogical reasons, here we only present the phase diagrams in the $\delta$ - $d$ space, which are in practice translated from the phase diagrams in the chemical potential space (see the example in Sec. $\mathrm{VB}$ for more details regarding the mapping between the two types of diagrams.)

\section{Without Feshbach Coupling $(g=0)$}

In the collisionless limit and without the Feshbach coupling $(g=0)$, our system simplifies to a three-species ideal gas model, where Eqs. (11) reduces to

$$
\mu_{i} \psi_{i}=0
$$

Figure1(a) is the corresponding phase diagram in the $\delta$ - $d$ space. This model is simple enough that the features in Fig. 1(a) are almost self-explanatory. Take as an example the doubly mixed phase ASF1-MSF with $\psi_{1} \neq 0$, $\psi_{3} \neq 0$, and $\psi_{2}=0$. Such a phase is possible only when $\mu_{1}=\mu_{3}=0$ but $\mu_{2}$ is arbitrary according to Eqs. (14). This means that $d=\psi_{1}^{2}-\psi_{2}^{2}=\psi_{1}^{2}>0$ and $\delta=\mu_{1}+\mu_{2}-\mu_{3}=\mu_{2}<0$, where the inequality is due to the stability criterion (13a). This is why the top left region of Fig. 1(a) hosts ASF1-MSF. Other features can be similarly explained. We note that Fig. 1(a) has similar properties as in Fig. 5 of Ref. [25]. The physics is very clear: as the Feshbach detuning is tuned from positive to negative or equivalently as the molecular energy level is lowered from above to below the atomic levels, the system makes a transition from the atomic side with atomic phases to the molecular side dominated with phases involving the molecular component. 

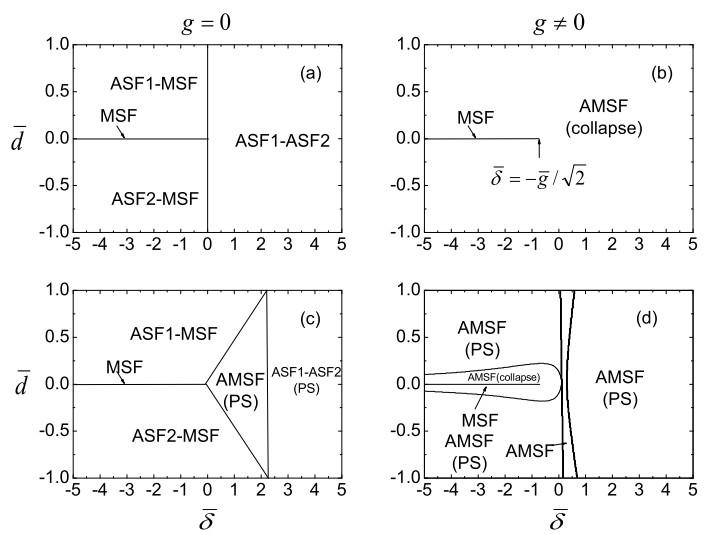

FIG. 1: The phase diagrams in the $\delta$ - $d$ space where (a) and (b) are produced with $\bar{g}=0$ for (a) and $\bar{g}=1$ for (b), and (c) and (d) correspond respectively to (a) and (b) while collisional coefficients are set at $\bar{\chi}_{11}=0.2, \bar{\chi}_{22}=0.3, \bar{\chi}_{12}=2$, and $\bar{\chi}_{3 i}=0$. See the text for units. For the sake of clarity, the labels for ASF1 and ASF2, which are located at $\bar{d}=1$ and $\bar{d}=-1$, respectively, are omitted.

\section{With finite Feshbach Coupling $(g \neq 0)$}

Figure 1(b) is the phase diagram in the $\delta$ - $d$ space, when the Feshbach coupling is turned on. Noticeable changes, compared to Fig. 1(a) where $g=0$, are the disappearance of the doubly mixed phases into the AMSF phase, and the corresponding transition boundaries into an atom-molecule crossover region (see Sec. IVA for the explanation). In addition, the MSF transition is shifted to $\bar{\delta}=-\bar{g} / \sqrt{2}$, owing to the modification of the molecular binding energy by the Feshbach coupling [26, 27, 30].

We explain below that without collisions, the AMSF phase will collapse. Let us begin with Eqs. (11) which, in the absence of collisions, reduce to $2 \mu_{1} \psi_{1}=-g \psi_{2} \psi_{3}$, $2 \mu_{2} \psi_{2}=-g \psi_{1} \psi_{3}, 2 \mu_{3} \psi_{3}=-g \psi_{1} \psi_{2}$. Thus, within the chemical potential space defined by

$$
\mu_{1}<0, \mu_{2}<0, \mu_{3}<0\left(\text { or } \mu_{1}+\mu_{2}<\delta\right),
$$

there exists a physical AMSF solution $\left(\psi_{i}>0\right)$ with a density distribution

$$
n_{1}=\frac{4 \mu_{2} \mu_{3}}{g^{2}}, n_{2}=\frac{4 \mu_{1} \mu_{3}}{g^{2}}, n_{3}=\frac{4 \mu_{1} \mu_{2}}{g^{2}} .
$$

This AMSF phase is unstable because the left-hand side of the stability condition (13c) is found to be $32 \mu_{1} \mu_{2} \mu_{3}$, which turns out to be less than zero as a result of the condition (15). The only other homogeneous solution besides this AMSF is the vacuum, which is stable within the space defined by Eq. (15) according to Eq. (13a). Thus, we conclude that the only route for this unstable AMSF to take is to collapse. The system may, however, be stabilized by kinetic energy without going into a complete collapse, exhibiting the so-called rarified liquid-like behavior [31]. The dynamics of phase separation and collapse may lead to quite complex spatial patterns [32].

\section{B. Collisions Involving Atoms Only}

In general, collisions greatly complicate the construction and understanding of the phase diagrams, because Eqs. (11) then become highly nonlinear and may support several AMSFs. In light of the fact that the collisional coefficients involving the molecular state are not known in most experiments, we ignore in this subsection all the collisions involving the molecular component $\left(\chi_{i 3}=0\right)$, and take into consideration only the atomic collisions. In particular, we focus our discussion on the case with $\bar{\chi}_{11}=0.2$ and $\bar{\chi}_{22}=0.3$, and $\bar{\chi}_{12}=2$, where the interspecies collision is far stronger than the intraspecies collisions.

\section{Without Feshbach Coupling $(g=0)$}

Figure1(c) is the phase diagram in the $\delta$ - $d$ space when $g=0$. Figure 1(c) contains, in addition to all the phases occurred in Fig. 1(a) where collisions are turned off, a unique AMSF given by

$$
n_{1}=\frac{\chi_{12} \mu_{2}-\chi_{22} \mu_{1}}{\chi_{12}^{2}-\chi_{11} \chi_{22}}, n_{2}=\frac{\chi_{12} \mu_{1}-\chi_{11} \mu_{2}}{\chi_{12}^{2}-\chi_{11} \chi_{22}}
$$

$n_{3}=\left(1-n_{1}-n_{2}\right) / 2$, and $\mu_{3}=0$. This AMSF is surrounded by three lines,

$$
\begin{aligned}
& d=-\frac{\delta}{\chi_{12}+\chi_{22}} \\
& d=\frac{\delta}{\chi_{12}+\chi_{11}} \\
& d=\frac{2 \delta-\chi_{11}-2 \chi_{12}-\chi_{22}}{\chi_{11}-\chi_{22}}
\end{aligned}
$$

serving as the borders between AMSF and MSF, ASF1MSF, and ASF1-ASF2, respectively. These equations are obtained from the conditions that $n_{i}=0(i=1,2,3)$, respectively. Evidently, all the lines are collapsed into a vertical line at $\delta=0$ and no AMSF is possible in the absence of collisions as in Fig. 1(a). Understandably, ASF1-ASF2 and its next level of hierarchy, AMSF, are both expected to be unstable against phase separation due to the strong repulsion between the two atomic components [8, 24].

\section{With finite Feshbach Coupling $(g \neq 0)$}

In the case of finite Feshbach coupling, we first obtain from Eqs. (11) that

$$
\psi_{3}=-\frac{g}{2 \mu_{3}} \psi_{1} \psi_{2}
$$


which, when substituted into the other two equations, gives a unique AMSF solution with the following atom density distribution

$$
n_{1}=\frac{\chi_{22} \mu_{1}-\chi_{12}^{\prime} \mu_{2}}{\chi_{11} \chi_{22}-\chi_{12}^{\prime 2}}, n_{2}=\frac{\chi_{11} \mu_{2}-\chi_{12}^{\prime} \mu_{1}}{\chi_{11} \chi_{22}-\chi_{12}^{\prime 2}}
$$

where $\chi_{12}^{\prime}=\chi_{12}+g^{2} / 4 \mu_{3}$ plays the role of an effective interaction between the two atomic species. The extra term $g^{2} / 4 \mu_{3}$ can be traced to the Feshbach coupling; an atom of species 1 appears to interact with an atom of species 2 via the molecular state by the Feshbach coupling of the strength $g$. This interaction becomes virtual when $\delta$ is large but can become substantial when $\delta$ is near resonance. Unlike the AMSF in the case of $g=0$, where $\mu_{3}=0, \mu_{3}$ here must be less than zero $\left(\mu_{3}<0\right)$. Thus, we always have $\chi_{12}^{\prime}<\chi_{12}$. It is well known that a double BEC mixture is stable when $\chi_{12}^{\prime 2}<\chi_{11} \chi_{22}$, and otherwise, it is unstable and will separate when $\chi_{12}^{\prime}>0$, and collapse when $\chi_{12}^{\prime}<0[8,24]$.

Figure 1(d) shows the phase diagram in the $\delta$ - $d$ space when $g$ is finite. For the reason given previously, we observe that the regions of doubly mixed phases give away to the AMSF crossover region. With our choice of $\chi_{i j}$, the system has a natural tendency to phase separate. However, we see that a narrow strip of stable AMSF emerges from the region near the Feshbach resonance. This is possible because $\chi_{12}^{\prime}$ can be substantially smaller than $\chi_{12}$ while remaining positive. This illustrates how one can change the mixture from immiscible to miscible by varying the Feshbach detuning [21].

In addition, in the region around the MSF line, because most populations will accumulate in the molecular component, $\mu_{3}=-g \psi_{1} \psi_{2} / 2 \psi_{3}$ will take a small negative value, which in turn makes the contribution from $g^{2} / 4 \mu_{3}$ to the effective interparticle interaction $\chi_{12}^{\prime}$ relatively strong. So the system will collapse in this region but phase separate outside this region where the repulsive interaction become dominant again.

\section{Collisions Involving Both Atoms and Molecules}

The main new feature when collisions involving molecules are also included is that more than one AMSFs may emerge. To demonstrate this feature, we consider, for simplicity, a model in which a finite $\chi_{33}$ is introduced in addition to the atomic collisional terms (while still keeping $\chi_{13}=\chi_{23}=0$ ). By solving Eqs. (11), we find that the AMSF phase is governed by equations same as Eqs. (18) and (19) except that $\mu_{3}$ is now replaced with $\mu_{3}^{\prime}=\mu_{3}-\chi_{33} n_{3}$. As can be seen, the chemical potential is now modulated by a Kerr nonlinear term $-\chi_{33} n_{3}$. It is well known that the Kerr nonlinear term can lead to multiple solutions that can result in multi-stability in nonlinear systems. Here, we find (not shown) that $n_{3}$ is governed by a fifth-order polynomial equation, and hence may support more than one physical solutions.

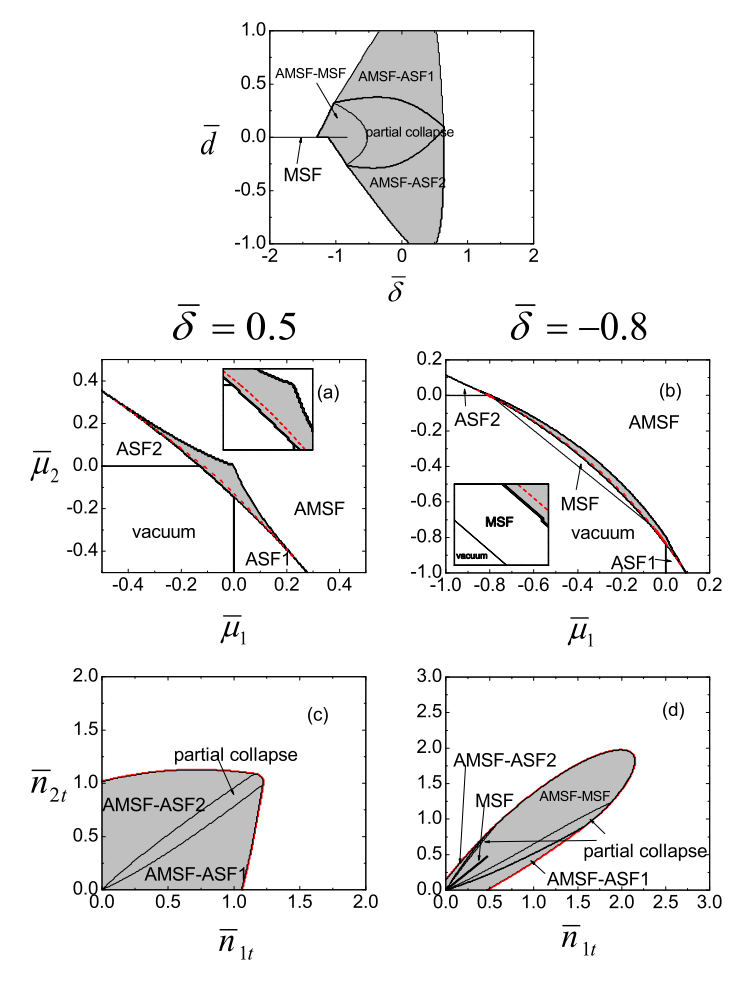

FIG. 2: (Color online) The phase diagrams in the $\delta-d$ space (the upmost figure, with total density $\bar{n}_{t}=1$ ), the chemical potential space [(a) and (b)], and the density space [(c) and (d)] when $\bar{g}=1$, $\bar{\chi}_{11}=0.2, \bar{\chi}_{22}=0.3, \bar{\chi}_{33}=0.245, \bar{\chi}_{12}=0.1225$, and $\bar{\chi}_{13}=$ $\bar{\chi}_{23}=0$. Additionally, (a) and (c) are produced with $\bar{\delta}=0.5$ while (b) and (d) are produced with $\bar{\delta}=-0.8$. The gray area in (a) and (b) represents the region where two stable phases can coexist. The red dashed lines inside the gray area are the first-order phase transition lines at which the ground state changes from one phase to the other. See the inset for more details of the region around the first-order line. Consult the text for units and for a detailed explanation of each diagram.

\section{One Stable AMSF and One Unstable AMSF}

Let us consider here a case where we choose the molecule-molecule collisional coefficient to be $\bar{\chi}_{33}=$ 0.245 , and all the atomic collisional parameters same as in Figs. 1(c) and (d) except that the interspecies repulsion is now set at a moderate value of $\bar{\chi}_{12}=0.1225$. The phase diagram in the $\delta$ - $d$ space for this case is displayed in the upmost figure of Fig. 2, which is all occupied by the AMSF state except for the line at $d=0$ marked with MSF. Because of the much reduced $\chi_{12}$, the system has a natural tendency to be in a miscible state and the AMSF will not phase separate (the white area) except in a small parameter region (the grey area) close to the Feshbach resonance. Unlike in Fig. 1 1 where we continue to label the phase separated region as AMSF, in Fig. 2] we will not show the unstable AMSF, instead, we divide it into 
regions and label them according to how the homogeneous (but unstable) AMSF is going to phase separate. For example, AMSF-ASF1 means that the homogeneous AMSF within the grey region of that label will phase separate into one AMSF and one ASF1.

The origin of this rich set of phase separations can be best understood from the chemical potential and density space. Consider, for example, $\bar{\delta}=0.5$. In Fig. [2(a), we construct the phase diagram in the chemical potential space. For a fixed set of chemical potentials, the coupled equations (11) are then found to support two physical AMSFs in some parameter regimes: one is stable and the other is unstable. Only the stable AMSF is shown here. As Fig. 2(a) illustrates, the stable AMSF coexists and shares the first-order transition line, respectively, with ASF1, ASF2, and vacuum. The first-order transition line in Fig. 2(a) is transformed into the grey region in Fig. 2(c) when the phase diagram is mapped from the chemical potential to the density space [33, 34]. For our purpose, we find it convenient to define the densities $n_{1 t}=n_{1}+n_{3}$ and $n_{2 t}=n_{2}+n_{3}$ so that $n_{1 t}+n_{2 t}$ and $n_{1 t}-n_{2 t}(=d)$ represent the total density and the population imbalance, respectively. For a system operating within the grey region in Fig. 2(c), it will phase separate into AMSF and ASF1, or AMSF and ASF2, and or AMSF and vacuum. The separated phases in each set have different densities, and phase separation is a natural course for a system to take in order to maintain the average densities specified within the respective domain of the phase separation. The phase separation into AMSFvacuum signals collapse.

As $\delta$ is reduced, for example, to $\bar{\delta}=-0.8$, a new phase separation, AMSF-MSF, appears in the upmost figure, which is again supported by Figs. 2(b) and (d) in the chemical potential and density space. Compared to $\bar{\delta}=$ 0.5 where the AMSF is unstable irrespective of the value of $d$, the AMSF at $\bar{\delta}=-0.8$ becomes a stable one when the population imbalance between the two atomic species is sufficiently large.

\section{Two Stable AMSFs and One Unstable AMSF}

The nonlinearity of Eqs. (11) may provide the opportunity for an unstable homogeneous AMSF to phase separate into two different AMSFs. Consider the model that shares the same parameters as in Fig. 2 except that $\chi_{12}$ is set back to a value $\left(\bar{\chi}_{12}=2\right)$ same as in Fig. 1. It is found that Eqs. (11) support three physical AMSF solutions, two of which satisfy the stability conditions (13) and are hence stable. The phase diagrams corresponding to this model are shown in Fig. 3. A coexistence region between two stable AMSFs having different population distributions is identified from the chemical potential space both for $\bar{\delta}=1$ [Fig. 3(a)] and $\bar{\delta}=0.5$ [Fig. 3(b)]. However, in experiments where the total population is fixed, AMSF1AMSF2 is accessible only to sufficiently small Feshbach detunings, for example, $\bar{\delta}=0.5$ (see the phases crossed
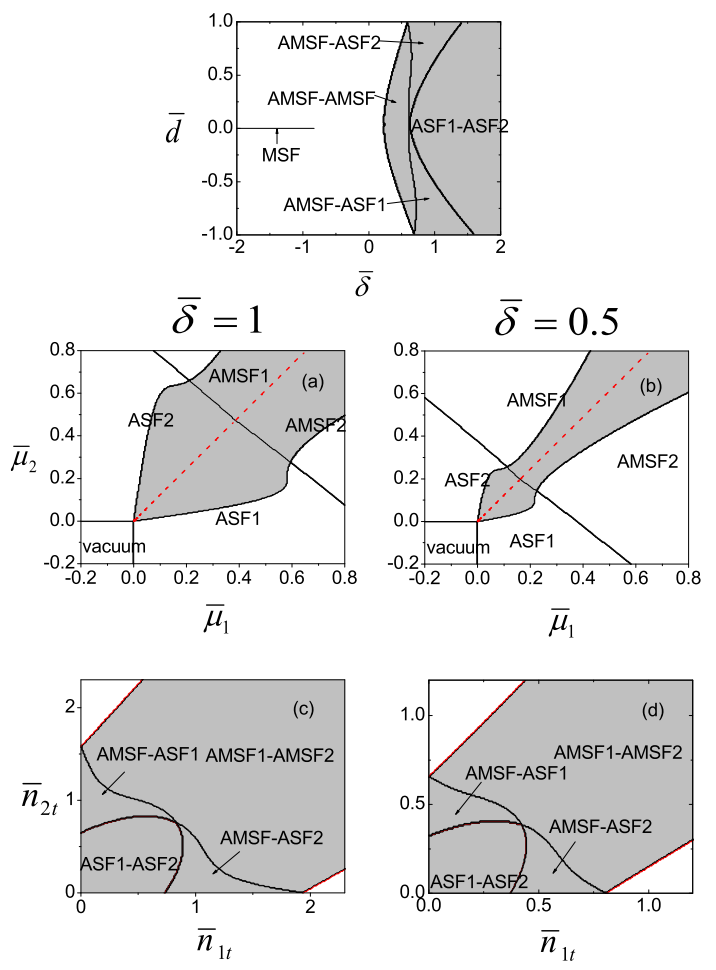

FIG. 3: (Color online) The phase diagrams similarly defined as in Fig. 2. The parameters are the same as those in Fig. 2 except that $\bar{\chi}_{12}=2$. Additionally, (a) and (c) are produced with $\bar{\delta}=1$ while (b) and (d) are produced with $\bar{\delta}=0.5$. The red dashed lines have the same meanings as that in Fig. 2. Consult the text for units.

by the (imagined) line $\bar{n}_{1 t}+\bar{n}_{2 t}=1$ in both Fig. [3) (c) and Fig. 3(d)]. We emphasize that so far, we have not seen reports of phase separation into two AMSFs both in theory and in experiments. In particular, we mention that the homonuclear systems are known to only support phase separations involving one AMSF [26]. For this reason, we suspect that this may be a phenomenon quite unique to the heteronuclear model.

This case also provides an example for us to see the effect of the molecule-molecule interaction on the stability of the AMSF. Compared to Fig. 11(d) where $\chi_{33}=0$, the upmost diagram of Fig. 3 shows that the presence of $\chi_{33}$ stabilized the AMSF on the molecular side of the Feshbach resonance.

\section{SUMMARY}

In conclusion, we have studied the zero-temperature mean-field phase diagrams of two-species atomic BEC mixtures with the interspecies Feshbach resonance under a variety of conditions, with a special attention being given to the subject of phase separation. We have 
found that on one hand, the Feshbach coupling alone cannot create a stable AMSF; to create stable AMSF, collisions must be present, and on the other hand, the Feshbach coupling may stabilize a system that has a natural tendency to phase separate under collisions. We have shown that the molecule-molecule collision can stabilize the AMSF on the molecular side of the Feshbach resonance. We have identified the interspecies Feshbach interaction to be the cause for the absence of the doubly mixed phases, as well as the reason for the presence of both pure atom and pure molecule phases in our model. This provides the opportunity, as we have indeed verified numerically, for observing the phase separation not only between AMSF and MSF but also those between AMSF and pure ASFs. We have also studied the collapse or partial collapse of the AMSF as special phase separations where one of the separated components is the vacuum. Under certain conditions, we have even found that our system is able to phase separate into two distinct AMSFs, which, we speculate, is a property unique to the heteronuclear model.

\section{Acknowledgments}

This work is supported by the National Natural Science Foundation of China under Grant No. 10588402 and
No. 10474055, the National Basic Research Program of China (973 Program) under Grant No. 2006CB921104, the Science and Technology Commission of Shanghai Municipality under Grant No. 06JC14026 and No. 05PJ14038, the Program of Shanghai Subject Chief Scientist under Grant No. 08XD14017, the Program for Changjiang Scholars and Innovative Research Team in University, Shanghai Leading Academic Discipline Project under Grant No. B480, the Research Fund for the Doctoral Program of Higher Education under Grant No. 20040003101 (W.Z.), by China Postdoctoral Science Foundation under Grant No. 44021570 (L.Z.) and by the US National Science Foundation (H.P., H.Y.L.), the US Army Research Office (H.Y.L.), and the Robert A. Welch Foundation (Grant No. C-1669), and the W. M. Keck Foundation (H.P.).

$\dagger^{\dagger}$ wpzhang@phy.ecnu.edu.cn

*ling@rowan.edu

**hpu@rice.edu
[1] C. J. Myatt, E. A. Burt, R. W. Ghrist, E. A. Cornell, and C. E. Wieman, Phys. Rev. Lett. 78, 586 (1997).

[2] D. S. Hall, M. R. Matthews, J. R. Ensher, C. E. Wieman, and E. A. Cornell, Phys. Rev. Lett. 81, 1539 (1998).

[3] J. Stenger, S. Inouye, D. M. Stamper-Kurn, H.-J. Miesner, A. P. Chikkatur, and W. Ketterle, Nature (London) 396, 345 (1998).

[4] T. L. Ho and V. B. Shenoy, Phys. Rev. Lett. 77, 3276 (1996).

[5] P. Ao and S. T. Chui, Phys. Rev. A 58, 4836 (1998).

[6] B. D. Esry, C. H. Greene, J. P. Burke, Jr., and J. L. Bohn, Phys. Rev. Lett. 78, 3594 (1997).

[7] H. Pu and N. P. Bigelow, Phys. Rev. Lett. 80, 1130 (1998); 80, 1134 (1998).

[8] E. Timmermans, Phys. Rev. Lett. 81, 5718 (1998).

[9] M. W. Zwierlein, A. Schirotzek, C. H. Schunck, and W. Ketterle, Science 311, 492 (2006).

[10] G. B. Partridge, W. Li, R. I. Kamar, Y. A. Liao, and R. G. Hulet, Science 311, 503 (2006); G. B. Partridge, W. Li, Y. A. Liao, R. G. Hulet, M. Haque, and H. T. C. Stoof, Phys. Rev. Lett. 97, 190407 (2006).

[11] D. DeMille, Phys. Rev. Lett. 88, 067901 (2002).

[12] A. Micheli, G. K. Brennen, and P. Zoller, Nature Phys. 2, 341 (2006).

[13] M. G. Kozlov, and L. N. Labzowsky, J. Phys. B 28, 1933 (1995).

[14] J. J. Hudson, B. E. Sauer, M. R. Tarbutt, and E. A. Hinds, Phys. Rev. Lett. 89, 023003 (2002).

[15] D. DeMille, S. Sainis, J. Sage, T. Bergeman, S. Kotochigova, and E. Tiesinga, Phys. Rev. Lett. 100, 043202
(2008).

[16] V. V. Flambaum, M. G. Kozlov, Phys. Rev. Lett. 99, 150801 (2007).

[17] T. Zelevinsky, S. Kotochigova, and J. Ye, Phys. Rev. Lett. 100, 043201 (2008).

[18] D. DeMille, S. B. Cahn, D. Murphree, D. A. Rahmlow, and M. G. Kozlov, Phys. Rev. Lett. 100, 023003 (2008)

[19] J. Doyle, B. Friedrich, R. V. Krems and F. MasnouSeeuws, Eur. Phys. J. D 31, 149 (2004); J. M. Hutson and P. Soldán, Int. Rev. Phys. Chem. 25, 497 (2006).

[20] G. Thalhammer, G. Barontini, L. De Sarlo, J. Catani, F. Minardi, and M. Inguscio, Phys. Rev. Lett. 100, 210402 (2008).

[21] S. B. Papp, J. M. Pino, and C. E. Wieman, Phys. Rev. Lett. 101, 040402 (2008).

[22] L. Zhou, W. Zhang, H. Y. Ling, L. Jiang, and H. Pu, Phys. Rev. A 75, 043603 (2007).

[23] M. Duncan, A. Foerster, J. Links, E. Mattei, N. Oelkers, and A. P. Tonel, Nucl. Phys. B 767, 227 (2007).

[24] D. C. Roberts and M. Ueda, Phys. Rev. A 73, 053611 (2006).

[25] T. Nishimura, A. Matsumoto, and H. Yabu, Phys. Rev. A 77, 063612 (2008).

[26] L. Radzihovsky, J. Park, and P. B. Weichman, Phys. Rev. Lett. 92, 160402 (2004).

[27] M. W. J. Romans, R. A. Duine, S. Sachdev, and H. T. C. Stoof, Phys. Rev. Lett. 93, 020405 (2004).

[28] L. Radzihovsky, P. B. Weichman, and J. I. Park, Annals of Physics 323, 2376 (2008).

[29] A. Simoni, M. Zaccanti, C. D'Errico, M. Fattori, G. 
Roati, M. Inguscio, and G. Modugno, Phys. Rev. A 77 , 052705 (2008).

[30] R. A. Duine and H. T. C. Stoof, Physics Reports 396 , 115 (2004).

[31] E. Timmermans, P. Tommasini, R. Côté, M. Hussein, and A. Kerman, Phys. Rev. Lett. 83, 2691 (1999); E. Timmermans, P. Tommasini, M. Hussein, and A. Kerman, Phys. Rep. 315, 199 (1999).
[32] S. Ronen, J. L. Bohn, L. E. Halmo, M. Edwards, arXiv:0807.4980

[33] D. E. Sheehy and L. Radzihovsky, Annals of Physics 322, 1790 (2007).

[34] F. M. Marchetti, C. Mathy, M. M. Parish, and D. A. Huse, Phys. Rev. B 78, 134517 (2008). 